International Journal of Applied Mathematical Research, 6 (4) (2017) $141-146$
International Journal of Applied Mathematical Research
WPC
Website: www.sciencepubco.com/index.php/IJAMR
doi: $10.14419 /$ ijamr.v6i4.8490
Research paper

\title{
Numerical comparison of nonstandard schemes for the Airy equation
}

\author{
Oluwaseye Adekanye $^{1 *}$ and Talitha Washington ${ }^{2}$ \\ ${ }^{1}$ Department of Mathematics, Howard University, Washington, DC, USA \\ ${ }^{2}$ Department of Mathematics, Howard University, Washington, DC, USA \\ *seyeadekanye@gmail.com
}

\begin{abstract}
This paper considers the Airy ordinary differential equation (ODE) and different ways it can be discretized. We first consider a standard discretization using the central difference scheme. We then consider two difference schemes which were created using a nonstandard methodology. Finally, we compare the different schemes and how well they approximate solutions to the Airy ODE.
\end{abstract}

Keywords: ODE; Nonstandard Finite Difference Scheme; Airy Equation

\section{Introduction}

In this paper, we will numerically compare various finite difference schemes for the Airy equation. The Airy equation is an ordinary differential equation (ODE) given by

$$
\frac{d^{2} y}{d x^{2}}-x y=0
$$

Equation (1) does not have a closed form solution.

Standard finite difference (SFD) schemes, such as the forward Euler scheme and the central difference scheme, can be used to numerically approximate the solutions to differential equations. The problem with these numerical schemes is that they often have numerical instabilities that depend on step size. A nonstandard finite difference (NSFD) scheme is a difference scheme which seeks to avoid numerical instabilities by incorporating dynamics of the system into the scheme. Dynamical consistency is achieved when the difference equation has the same qualitative properties as its corresponding differential equation. To create a suitable NSFD scheme for a differential equation, a set of modeling strategies can be used to optimize dynamical consistency [2]. Some of these strategies are as follows:

1. Exact finite-difference schemes generally require that nonlinear terms be modeled nonlocally [6].

2. The discrete derivatives for differential equations have denominator functions $\varphi(h)$ that are typically more complicated than those used in the standard modeling procedure. For example, the denominator function $\varphi(h)$ of a first order differential equation has the property $\varphi(h)=h+\mathscr{O}\left(h^{2}\right)$. This allows for the construction of a larger class of finite difference models and also provides for more ambiguity in the modeling process $[2,4]$.
3. The order of the discrete derivatives in exact finite difference schemes is always equal to the corresponding order of the derivatives of the differential equation.

4. Important properties of the differential equations and/or their solutions should be incorporated into their corresponding discrete forms. Properties such as positivity of solution, traveling wave solutions, periodic solutions, etc., should be preserved, if possible.

These modeling strategies have become the basis for creating NSFD schemes.

We will focus on comparing the NSFD scheme in [2] known as the Mickens-Ramadhani scheme with the NSFD scheme in [8]. For ease of reference, we will call the NSFD scheme in [8] the Yaghoubi-Najafi scheme. For our numerical comparisons, the following initial conditions were used.

$$
y_{0}=1, \quad y_{0}^{\prime}=1
$$

This paper is ordered as follows. In section 2, we will introduce the power series solution of equation (1). Next in section 3, we will discuss and compare the standard scheme Euler's method, the Yaghoubi-Najafi scheme, and the Mickens-ramadhani scheme. In section 4 we will numerically compare the schemes and investigate each scheme's performance via numerical experiments. Finally, in section 5, we will discuss the results and future work.

\section{Power Series Solution}

The Airy equation (1) does not have a closed form solution. However, its solution can be represented by a power series. First assume $y$ has a power series expansion, i.e., $y(x)=\sum_{n=0}^{\infty} a_{n} x^{n}$. Substituting this into equation (1) gives

$$
\sum_{n=2}^{\infty} a_{n} x^{n-2}-\sum_{n=0}^{\infty} a_{n} x^{n+1}=0 \text {. }
$$


After some algebraic manipulations, the coefficients must satisfy the following recurrence relations.

$$
\begin{aligned}
a_{2} & =0, \\
a_{n+2} & =\frac{a_{n-1}}{(n+2)(n+1)}, \quad \text { for } n=1,2, \ldots .
\end{aligned}
$$

From the recurrence relations in equations (4)-(5), we have

$$
\begin{aligned}
a_{3 k+2} & =0, & & \text { for } k=0,1,2, \ldots, \\
a_{3 k} & =\frac{a_{0}}{(2 \cdot 3)(5 \cdot 6) \cdots((3 k-1)(3 k))}, & & \text { for } k=1,2,3, \ldots, \\
a_{3 k+1} & =\frac{a_{1}}{(3 \cdot 4)(6 \cdot 7) \cdots((3 k+1)(3 k))}, & & \text { for } k=1,2,3, \ldots
\end{aligned}
$$

Therefore the series solution to equation (1) is

$$
y(x)=\sum_{k=0}^{\infty} a_{3 k} x^{3 k}+\sum_{k=0}^{\infty} a_{3 k+1} x^{3 k+1}+\sum_{k=0}^{\infty} a_{3 k+2} x^{3 k+2}
$$

or

$$
\begin{aligned}
y(x)= & a_{0}\left[1+\sum_{k=1}^{\infty} \frac{x^{3 k}}{(2 \cdot 3)(5 \cdot 6) \cdots((3 k-1)(3 k))}\right] \\
& +a_{1}\left[x+\sum_{k=1}^{\infty} \frac{x^{3 k+1}}{(3 \cdot 4)(6 \cdot 7) \cdots((3 k+1)(3 k))}\right] .
\end{aligned}
$$

We will use the power series solution in equation (10) to numerically compare performance of the various schemes we will present in the next section.

\section{Development of Finite Difference Schemes}

This section focuses on the development of standard and nonstandard finite difference schemes for equation (1) with initial conditions given in (2).

\subsection{Standard Finite Difference Scheme}

A standard difference scheme for equation (1) can be derived by making the following substitutions.

$$
\begin{aligned}
y(x) & \rightarrow y_{k} \\
y(x+h) & \rightarrow y_{k+1} \\
y(x-h) & \rightarrow y_{k-1} \\
x & \rightarrow x_{k}
\end{aligned}
$$

The resulting standard finite difference scheme for equation (1) is

$$
\frac{y_{k+1}-2 y_{k}+y_{k-1}}{h^{2}}-x_{k} y_{k}=0 .
$$

Solving equation (15) explicitly for $y_{k+1}$ gives

$$
y_{k+1}=\left(h^{2} x_{k}+2\right) y_{k}-y_{k-1} \quad \text { for } k=1,2, \ldots .
$$

In equation (16), note that in order to compute the next time step, two previous values, $y_{0}$ and $y_{1}$ are required. Using the second order Taylor expansion, we get

$$
y_{1}=y_{0}+h y_{0}^{\prime}+\frac{h^{2}}{2 !} y_{0}^{\prime \prime}
$$

Making use of the initial conditions given in (2), we get the following.

$$
y_{1}=1+h
$$

Therefore, we are able to get the desired second value that will enable us to implement the standard scheme (16).

\subsection{Yaghoubi-Najafi Finite Difference Scheme}

In [8], Yaghoubi and Najafi have the following nonstandard scheme for equation (1)

$$
\frac{y_{k+1}-2 y_{k}+y_{k-1}}{\varphi(h)}-x_{k} y_{k}=0
$$

where the denominator function is given by

$$
\varphi(h)=4 \sin ^{2}\left(\frac{h}{2}\right) .
$$

Solving (19) for $y_{k+1}$ gives

$$
y_{k+1}=\left(2+\varphi(h) x_{k}\right) y_{k}-y_{k-1} .
$$

We will refer to equation (21) as the Yaghoubi-Najafi Scheme. Note that the denominator function given in equation (20) is the same denominator function put forth by Mickens in [2] for the exact finite difference scheme of the harmonic oscillator equation,

$$
\frac{d^{2} y}{d x^{2}}+y=0
$$

The scheme (21) also requires two initial values to compute the next time step value. It appears the authors of [8] did not make use of a standard method to compute the value for $y_{1}(x)$. However, they might have used a nonstandard method such as

$$
y_{1}=y_{0}+\varphi(h) y_{0}^{\prime} .
$$

The denominator function used to determine $y_{1}$ can be calculated the following way. From the initial conditions in equation (2),

$$
\frac{d y(0)}{d x}=y(0)=1 \text {. }
$$

Thus, we can assume that initially, the solution satisfies

$$
\frac{d y}{d x}=y
$$

which has the exact difference scheme [5]

$$
\frac{y_{k+1}-y_{k}}{e^{h}-1}=y_{k} \text {. }
$$

Therefore

$$
y_{1}=e^{h} y_{0}
$$

and using the initial condition from (1), we have

$$
y_{1}=e^{h} \text {. }
$$

In the numerical computation section, we will determine wether calculating the value of $y_{1}(x)$ in a nonstandard way gives better approximations than when the value of $y_{1}(x)$ is calculated in a standard way.

\subsection{Mickens-Ramadhani Finite Difference Scheme}

In this section, we will use Mickens' nonstandard methodology as discussed in section 1 to give the reader details on how to construct the scheme from [2]. We start by considering the harmonic oscillator equation,

$$
\frac{d^{2} y}{d t}+\lambda y=0
$$

Equation (28) can be written as a system of two coupled first order ODEs. That is, $u^{(1)}=\lambda y$ and $u^{(2)}=\lambda y^{\prime}$. Then

$$
\begin{aligned}
& \frac{d u^{(1)}}{d t}=u^{(2)} \\
& \frac{d u^{(2)}}{d t}=-u^{(1)},
\end{aligned}
$$


with initial conditions

$$
u_{0}^{(1)}=u^{(1)}\left(t_{0}\right), \quad u_{0}^{(2)}=u^{(2)}\left(t_{0}\right)
$$

The solutions are

$$
\begin{aligned}
& u^{(1)}(t)=C_{1} e^{i \sqrt{\lambda} t}+C_{2} e^{-i \sqrt{\lambda} t} \\
& u^{(2)}(t)=i \sqrt{\lambda} C_{1} e^{i \sqrt{\lambda} t}-i \sqrt{\lambda} C_{2} e^{-i \sqrt{\lambda} t} .
\end{aligned}
$$

Using the initial condition to solve for $C_{1}$ and $C_{2}$, equations (32) and (33) become

$$
\begin{aligned}
u^{(1)}(t) & =\left(\frac{1}{2} u^{(1)}\left(t_{0}\right)-\frac{i}{2 \sqrt{\lambda}} u^{(2)}\left(t_{0}\right)\right) e^{i \sqrt{\lambda}\left(t-t_{0}\right)} \\
& +\left(\frac{1}{2} u^{(1)}\left(t_{0}\right)+\frac{i}{2 \sqrt{\lambda}} u^{(2)}\left(t_{0}\right)\right) e^{-i \sqrt{\lambda}\left(t-t_{0}\right)} \\
u^{(2)}(t) & =i \sqrt{\lambda}\left(\frac{1}{2} u^{(1)}\left(t_{0}\right)-\frac{i}{2 \sqrt{\lambda}} u^{(2)}\left(t_{0}\right)\right) e^{i \sqrt{\lambda}\left(t-t_{0}\right)} \\
& -i \sqrt{\lambda}\left(\frac{1}{2} u^{(1)}\left(t_{0}\right)+\frac{i}{2 \sqrt{\lambda}} u^{(2)}\left(t_{0}\right)\right) e^{-i \sqrt{\lambda}\left(t-t_{0}\right)} .
\end{aligned}
$$

With the following substitutions,

$$
\begin{aligned}
u(t) & \rightarrow u_{k+1} \\
u\left(t_{0}\right) & \rightarrow u_{k} \\
t-t_{0} & \rightarrow h,
\end{aligned}
$$

equations (34) and (35) become

$$
\begin{aligned}
u_{k+1}^{(1)}= & {\left[\frac{1}{2} u_{k}^{(1)}-\frac{i}{2 \sqrt{\lambda}} u_{k}^{(2)}\right] e^{i \sqrt{\lambda} h} } \\
+ & {\left[\frac{1}{2} u_{k}^{(1)}+\frac{i}{2 \sqrt{\lambda}} u_{k}^{(2)}\right] e^{-i \sqrt{\lambda} h} } \\
u_{k+1}^{(2)} & =i \sqrt{\lambda}\left[\left(\frac{1}{2}\right) u_{k}^{(1)}-\left(\frac{1}{2 \sqrt{\lambda}} i\right) u_{k}^{(2)}\right] e^{i \sqrt{\lambda} h} \\
& -i \sqrt{\lambda}\left[\left(\frac{1}{2}\right) u_{k}^{(1)}+\left(\frac{1}{2 \sqrt{\lambda}} i\right) u_{k}^{(2)}\right] e^{-i \sqrt{\lambda} h} .
\end{aligned}
$$

Equations (39) and (40) can be rewritten using the identities

$$
e^{i \sqrt{\lambda} h}=\cos (\sqrt{\lambda} h)+i \sin (\sqrt{\lambda} h)
$$

and

$$
e^{-i \sqrt{\lambda} h}=\cos (\sqrt{\lambda} h)-i \sin (\sqrt{\lambda} h)
$$

This gives

$$
\begin{aligned}
& u_{k+1}^{(1)}=u_{k}^{(1)} \cos (\sqrt{\lambda} h)+\frac{1}{\sqrt{\lambda}} u_{k}^{(2)} \sin (\sqrt{\lambda} h) \\
& u_{k+1}^{(2)}=-\sqrt{\lambda} u_{k}^{(1)} \sin (\sqrt{\lambda} h)+u_{k}^{(2)} \cos (\sqrt{\lambda} h) .
\end{aligned}
$$

After some algebraic manipulations, we can eliminate the $u^{(2)}$ terms, drop the superscript notation, and obtain

$$
u_{k+1}-2 u_{k} \cos (\sqrt{\lambda} h)+u_{k-1}=0
$$

After some further manipulations of equation (43), we get a nonstandard scheme for equation (28) which is

$$
\frac{u_{k+1}-2 u_{k}+u_{k-1}}{\left(\frac{4}{\lambda}\right) \sin ^{2}\left(\frac{\sqrt{\lambda} h}{2}\right)}+\lambda u_{k}=0 .
$$

We will refer to equation (44) as the Micken-Ramadhani scheme for the harmonic oscillator equation. Note that equation (44) holds wether $\lambda$ is positive or negative. This result follows directly from the use of the relation [2]

$$
\sin (i \theta)=i \sinh (\theta)
$$

Replacing the constant $\lambda$ in equation (44) by the discrete representation of $f(x)=-x$, i.e.,

$$
\lambda \rightarrow-x_{k},
$$

gives the nonstandard finite difference representation for equation (1) [2]

$$
\frac{u_{k+1}-2 u_{k}+u_{k-1}}{\left(\frac{-4}{x_{k}}\right) \sin ^{2}\left(\frac{i \sqrt{x_{k}} h}{2}\right)}-x_{k} u_{k}=0 .
$$

We will refer to equation (47) as the Mickens-Ramadhani scheme for the Airy equation.

If $x_{k}>0$, using the relation (45) we obtain the following nonstandard difference scheme for equation (1)

$$
u_{k+1}=\left[2+4 \sinh ^{2}\left(\frac{\sqrt{x_{k}} h}{2}\right)\right] u_{k}-u_{k-1} .
$$

If $x_{k}<0$, using substitution we have the non-standard difference scheme for equation (1)

$$
u_{k+1}=2\left[\cos \left(h \sqrt{x_{k}}\right)\right] u_{k}-u_{k-1}
$$

If $x_{k}=0$, we have the following scheme for equation (1)

$$
u_{k+1}=2 u_{k}-u_{k-1}
$$

All three schemes (16), (21) and (48) discussed in this section require two data points to get started. From the initial conditions given in (2), we have the value for $y_{0}$. However the value for $y_{1}$ has to be calculated using the initial velocity $y_{1}^{\prime}=1$. In the next section, we will show that the way in which the second data point $y_{1}$ is calculated can have an effect on the difference schemes performance. We will show this by numerically comparing equations (16), (21) and (48) to see how close they are to the series solution given by equation (10).

\section{Numerical Results}

In this section, we display some numerical results for the MickensRamadhani scheme (48), Yaghoubi-Najafi scheme (21) and the standard central difference scheme (15) applied to the Airy Equation (1). We previously mentioned that equation (1), has no known analytical solution. However we will be using the power series solution developed in section 2 to determine how well the schemes perform. We will judge the performance of a difference scheme in two ways. The first is analyzing how close the numerical solutions to the difference equations are to the power series solution for varying step sizes. The second measure of performance will be derived by varying the step sizes and taking a max norm error at the last time value, between the power series solution and the difference scheme solution. For the first set of numerical approximations, each difference scheme were started of with the same initial conditions. That is, for equations (16), (21), and (48), the same value of $y_{0}$ and $y_{1}$ were used. Note that for $y_{1}$, the value of $y(1)$ from the series solution (10) was used. In figure 1 and figure 2, we see how the numerical solutions using equations (16), (21), and (48) behave compared to the series solution given by equation (10). Judging performance based on this, it appears that the Mickens-Ramadhani scheme (48) outperforms the other two schemes. With a step size of $h=0.25$ and $h=0.5$, figures 1 and 2 show that the Mickens-Ramadhani scheme approximations stays closer to the series solution curve than both the Yaghoubi-Najafi scheme approximations as well as the standard scheme approximations. Next we determine how well the MickensRamadhani scheme does compared to the other two. At $x=4$, the max norm error between the power series solution (10) and the difference scheme approximations was calculated. We noticed that as the step-size increased, the max norm error at $x=4$ increased 
as well. However as we can see in figure 3, the max norm error when the Mickens-Ramadhani scheme is used remains relatively low compared to the other two difference schemes. A log-log plot was generated to determine an approximate order of convergence of the difference scheme. In figure 4, we found the slope of the log-log plots generated to be of magnitude approximately equal to two. Therefore, we concluded that difference schemes were approximately second order accurate.

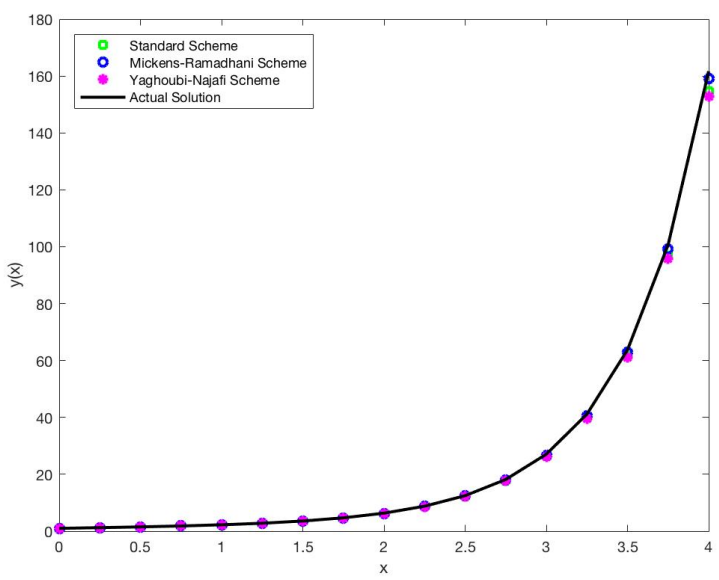

Figure 1: $h=0.25$

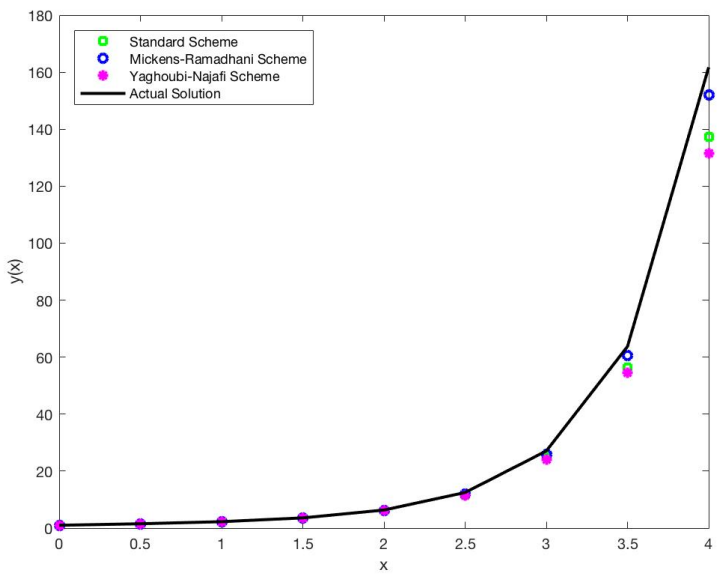

Figure 2: $h=0.5$

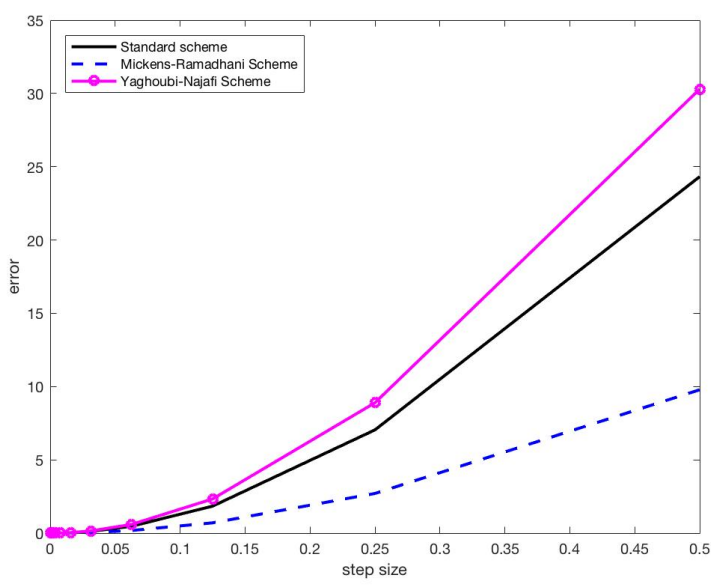

Figure 3: Graph of error of each numerical scheme with respect to the stepsize at $x=4$

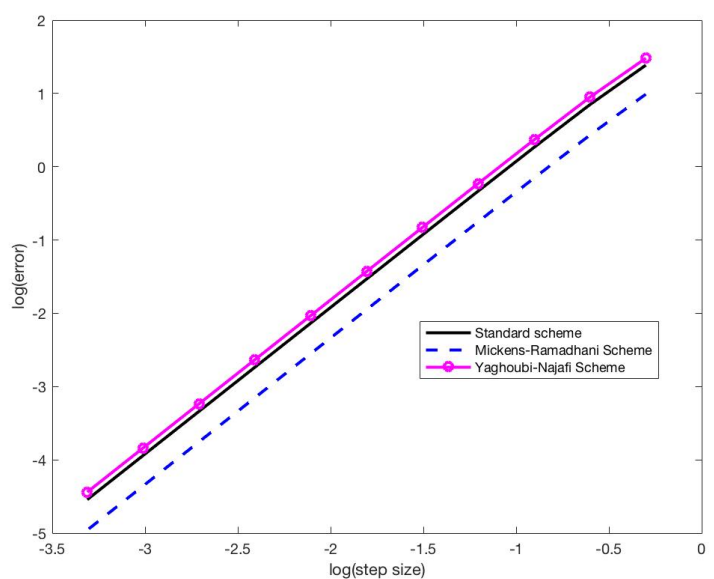

Figure 4: $\log -\log$ plot of error with respect to step-size at $x=4$

Now we will show the how using different values for $y_{1}$ affects the performance of the difference schemes. To do this, we calculated the value $y_{1}$ for each difference schemes in different ways. For the standard scheme, we used equation (18) to calculate the value for $y_{1}$. For the Yaghoubi-Najafi scheme (21), the equation (27) was used. Lastly for the Mickens-Ramhadi scheme (48), the following formula was used to calculate $y_{1}$.

$$
y_{1}=\sinh (h)+y_{0}
$$

In figures 5 and 6 , we see how the numerical solutions using equations (16), (21), and (48) behave compared to the series solution given by equation (10). It is difficult to determine which scheme performs best by referring to figures 5 and 6 alone because of the difficulty in seeing how close each schemes approximations are to the series solution curve. However using figure 7 , we can see that the Mickens-Ramadhani scheme (48) still outperforms the other two schemes since the magnitude of the error derived when using the Mickens-Ramadhani scheme is relatively less than the errors derived when using the other two schemes. This is with the exception of the step-size interval $0.25-0.35$ where the Yaghoubi-Najafi scheme performs the best. A log-log plot was generated to determine an approximate order of convergence of the difference scheme (see figure 8). In this case, the standard scheme and the MickensRamadhani scheme maintained their second order accuracy because the slope of the log-log plots generated were of magnitude approximately equal to two. However, the Yaghoubi-Najafi scheme appears 
to now be only first order accurate because the slope of the log-log plots generated to be of magnitude approximately equal to one.

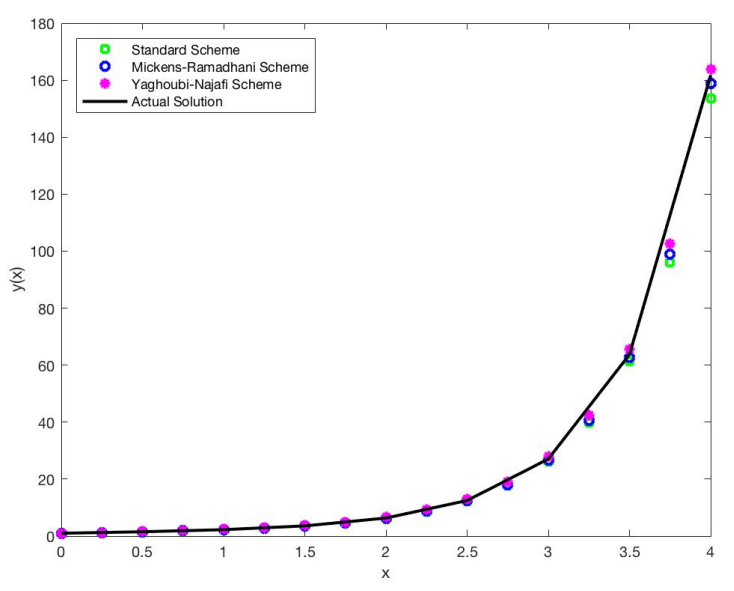

Figure 5: $h=0.25$

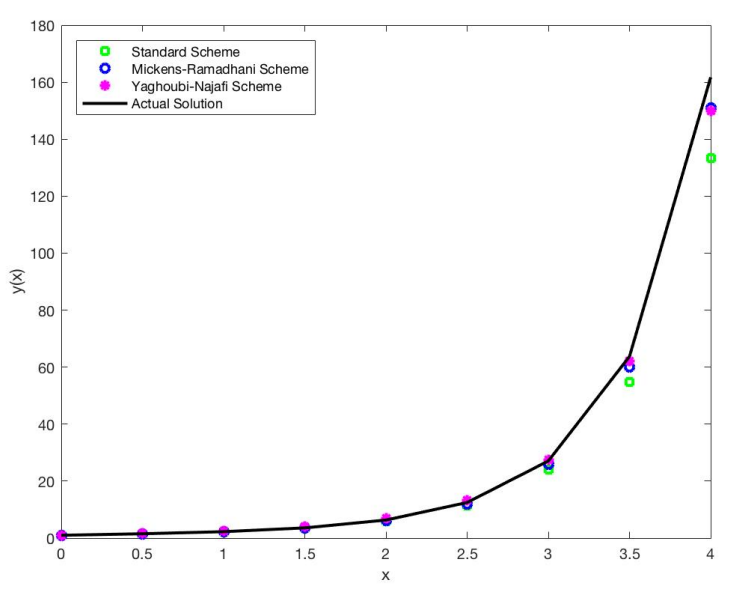

Figure 6: $h=0.5$

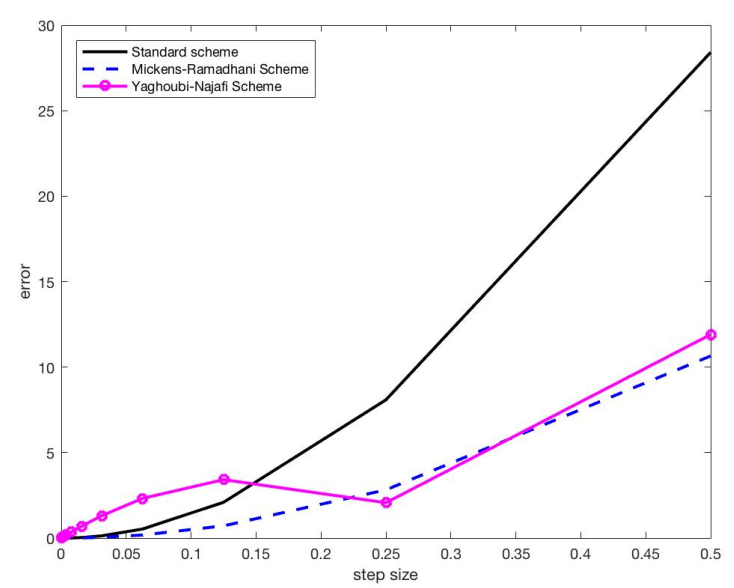

Figure 7: Graph of error of each numerical scheme with respect to the stepsize at $x=4$

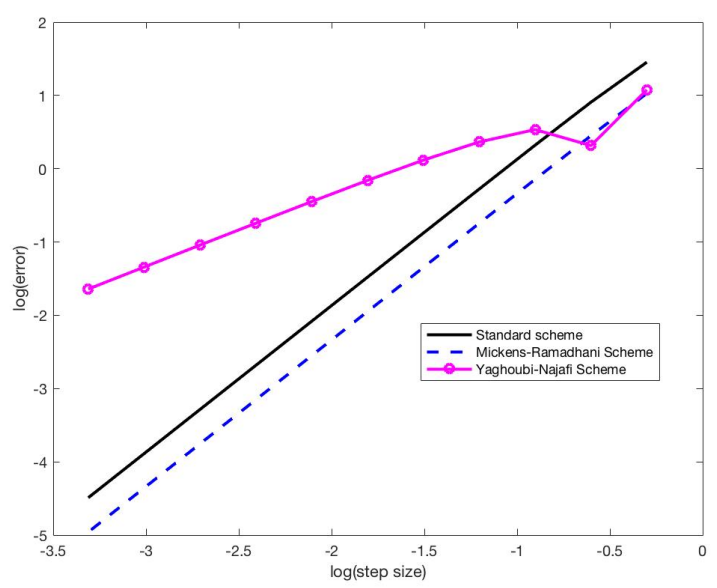

Figure 8: $\log -\log$ plot of error with respect to step-size at $x=4$

In some instances, the choice of the initial conditions can influence the numerical outputs. In figure 3 and figure 7, when the initial conditions used for the difference schemes $y_{0}$ and $y_{1}$ was the same as the first two data points in the series solution $y(1)$ and $y(2)$, the Mickens-Ramhadani scheme outperformed the other two schemes (figure 3). Also, when the value of $y_{1}$ was calculated based on the difference scheme in question, the Mickens-Ramhadani scheme still outperformed the other two schemes (see figure 7). However, calculating the value of $y_{1}$ in the nonstandard way (27) for the YaghoubiNajafi scheme appears to reduce the max norm error. So in this case, the choice in how the second initial condition is chosen has a significant impact on the performance of the scheme.

\section{Conclusion}

This paper compares different finite difference schemes for the Airy ordinary differential equation (1). It is important to find appropriate approximation methods for equations such as equation (1), which have no closed form solution. Due to the nonexistence of a closed form solution for equation (1), we made use of a series solution (10) in order to judge the performance of the finite difference schemes (16), (21), and (48).

To utilize the difference schemes discussed in this paper, two initial data points are required. In the case of this paper, one data point was given $y_{0}=1$ while the second had to be calculated using the initial condition $y_{0}^{\prime}=1$. This led us to investigate wether the method in which the second data point was calculated was of any consequence. We found that in the case of the standard finite difference scheme and the Yaghoubi-Najafi difference scheme, it did matter how the second data point was calculated. However we found that for the Micken-Ramadhani scheme, the behavior of the solutions remains somewhat consistent even when the method of calculating the second data point changed. We were able to numerically determine that overall, the Mickens-Ramadhani (48) outperformed both the standard difference scheme (16) and the other nonstandard difference scheme (21).

\section{References}

[1] L. Gr. Ixaru, M. Rizea, "Numerov method maximally adapted to the Schrödinger equation", J. Comput. Phys., Vol. 73, (1987), 306-324.

[2] R. E. Mickens, Nonstandard finite difference models of differential equations, World Scientific Publishing Co. Pte. Ltd., (1994).

[3] R. E. Mickens, "Nonstandard finite difference schemes for differential equations", Journal of Difference Equations and Applications, Vol. 8 $9,(2002), 823-847$.

[4] R. E. Mickens, A. Smith, "Finite-difference models of ordinary differential equations: influence of denominator functions", Journal of the Franklin Institute Vol. 327, (1990), 143-149. 
[5] R. E. Mickens, "Calculation of denominator functions for nonstandard finite difference schemes for differential equations satisfying a positivity condition", SIAM J. Math. Anal. Vol. 33, (2002), 672-691.

[6] R. E. Mickens, Advances in the application of nonstandard finite difference schemes World Scientific Publishing Co. Pte. Ltd. (2005).

[7] R. Chen, Z. Xu and L. Sun, "Finite-difference scheme to solve Schrödinger equations", Physical Review E Vol. 47, (1993), 3799 3802

[8] A. R. Yaghoubi, H. Saberi Najafi, "Comparison between standard and non-standard finite difference methods for solving first and second order ordinary differential equations", International Journal of Applied Mathematical Research Vol. 4, No. 2,(2015), 316-324. 